This item was submitted to Loughborough's Research Repository by the author.

Items in Figshare are protected by copyright, with all rights reserved, unless otherwise indicated.

\title{
Angular dependence of nonclassical magnetic quantum oscillations in a quasi-two-dimensional multiband Fermi liquid with impurities
}

PLEASE CITE THE PUBLISHED VERSION

http://link.aps.org/doi/10.1103/PhysRevB.65.035418

PUBLISHER

(C) The American Physical Society

\section{LICENCE}

CC BY-NC-ND 4.0

\section{REPOSITORY RECORD}

Bratkovsky, A.M., and A.S. Alexandrov. 2019. "Angular Dependence of Nonclassical Magnetic Quantum Oscillations in a Quasi-two-dimensional Multiband Fermi Liquid with Impurities". figshare. https://hdl.handle.net/2134/1158. 
This item was submitted to Loughborough's Institutional Repository (https://dspace.lboro.ac.uk/) by the author and is made available under the following Creative Commons Licence conditions.

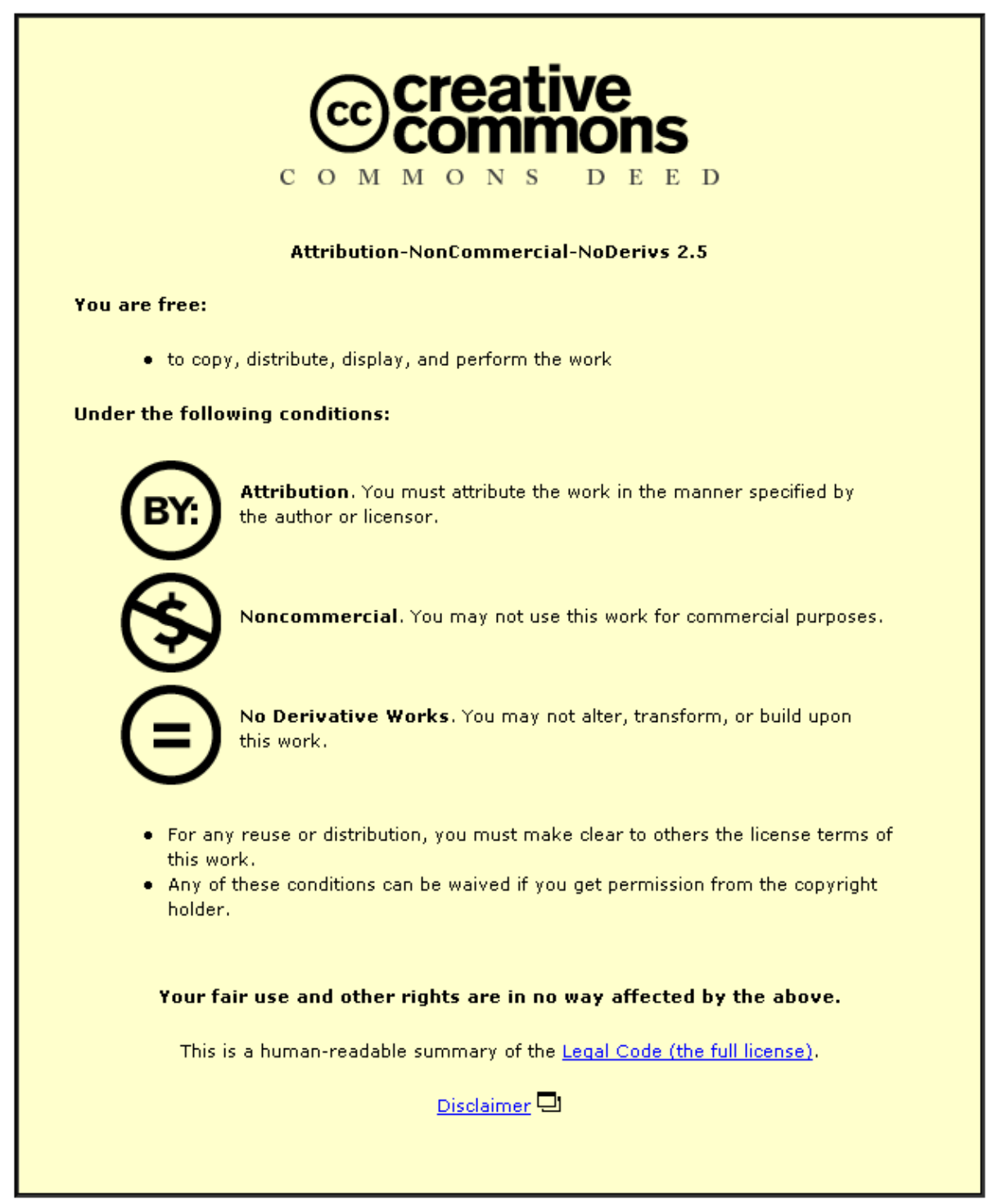

For the full text of this licence, please go to: http://creativecommons.org/licenses/by-nc-nd/2.5/ 


\title{
Angular dependence of novel magnetic quantum oscillations in a quasi-two-dimensional multiband Fermi liquid with impurities
}

\author{
A.M. Bratkovsky ${ }^{1}$ and A.S. Alexandrov ${ }^{2}$ \\ ${ }^{1}$ Hewlett-Packard Laboratories, 1501 Page Mill Road, Palo Alto, California 94304 \\ ${ }^{2}$ Department of Physics, Loughborough University, LE11 3TU, United Kingdom
}

(April 24, 2001)

\begin{abstract}
The semiclassical Lifshitz-Kosevich-type description is given for the angular dependence of quantum oscillations with combination frequencies in a multiband quasi-two-dimensional Fermi liquid with a constant number of electrons. The analytical expressions are found for the Dingle, thermal, spin, and amplitude (Yamaji) reduction factors of the novel combination harmonics, where the latter two strongly oscillate with the direction of the field. At the "magic" angles those factors reduce to the purely two-dimensional expressions given earlier. The combination harmonics are suppressed in the presence of the non-quantized ("background") states, and they decay exponentially faster with temperature and/or disorder compared to the standard harmonics, providing an additional tool for electronic structure determination. The theory is applied to $\mathrm{Sr}_{2} \mathrm{RuO}_{4}$.
\end{abstract}

71.18.+y, 71.27.+a, 73.21.-b, 73.90.+f

The low-dimensional organic conductors exhibit very unusual electronic properties, like magnetic field induced superconductivity, and are the focus of solid state research [1]. Magnetic oscillations of susceptibility and resistivity are the main source of detailed information about their electronic structure, yet there is no complete theory of these phenomena for quasi-two-dimensional (quasi-2D) metals. The analytical semiclassical (LifshitzKosevich type 22) theory of the de Haas-van Alphen combination harmonics in the multiband two-dimensional (2D) metals has been suggested recently [3]. Even earlier it was shown [4] for a system like $\mathrm{Sr}_{2} \mathrm{RuO}_{4}$ [5], that the magnetic quantum oscillations in a multiband $2 \mathrm{D}$ metal with a fixed electron density [canonical ensemble (CE)] are qualitatively different from those in an open system where the chemical potential is fixed [grand canonical ensemble (GCE)]. The chemical potential oscillates with the field in closed systems and this provides a mechanism for different bands to communicate with each other in CE producing a dHvA signal with the "sum" $\mid$ and the "difference" combination frequencies [6] in addition to the ordinary dHvA frequencies. Those novel oscillations have been observed in quantum well structures [9] and $\mathrm{Sr}_{2} \mathrm{RuO}_{4}$ 10,11. Their amplitudes are comparable with the standard components, and they are robust with respect to any background (non-quantized) density of states at low temperatures [6], but fall off exponentially faster with the temperature [3].

Many layered systems, including $\mathrm{Sr}_{2} \mathrm{RuO}_{4}$, are actually quasi-2D. Weak coupling between conducting layers, which introduces a dispersion of the Fermi surface in the direction perpendicular to the layers, leads to a strong dependence of magnetic oscillations on the angle $\theta$ between the normal to the conducting planes and the magnetic field $\vec{B}[12]$. This is related to the fact that the energy spectrum in the field becomes almost 2D (the width of the Landau minibands almost vanishes) at some "magic" (Yamaji) angles [13], where the amplitude of the oscillations is strongly enhanced. The theory [3] applies only to the clean systems at the magic angles. The full analytical semiclassical theory of the quasi-2D multiband canonical metals at finite temperature with full account for the angular dependence of the Landau miniband widths and the spin factors, the collision broadening of the minibands (Dingle factor), and the background density of states is given in the present paper and applied to a generic case of $\mathrm{Sr}_{2} \mathrm{RuO}_{4}$.

First, we derive a convenient expression for a multiband two-dimensional thermodynamic potential in magnetic field $B$, mainly in the units $\hbar=|e|=c=1$,

$$
\Omega=-T \int d \epsilon \mathcal{N}(\epsilon, B) \ln \left(1+\exp \frac{\mu-\epsilon}{T}\right)
$$

Consider the multiband quasi-2D system with a general dispersion law

$$
\epsilon=\Delta_{\alpha}+\frac{k_{x}^{2}+k_{y}^{2}}{2 m_{\alpha}}-2 t_{\alpha} \cos j k_{z} d,
$$

where $k_{x}, k_{y}$ are the momenta in the conducting plane, $t_{\alpha}$ is the hopping between the (conducting) layers, $d$ the distance between the layers, $j$ the integer number. We are mainly interested in the situation where the kinetic energy of electrons is much larger than the hopping between layers, $\mu-\Delta_{\alpha} \gg 2 t_{\alpha}$. The density of states (DOS) with an account for collision broadening of the Landau levels (Dingle factor) [15,16] can be written as

$$
\mathcal{N}(\epsilon, B)=\sum_{\alpha, b g}^{\text {bands,spin }} \mathcal{N}_{\alpha}(\epsilon, B),
$$




$$
\mathcal{N}_{\alpha}(\epsilon, B)=-\frac{1}{\pi} \operatorname{Im} \sum_{n=0}^{\infty} \frac{\Delta S}{(2 \pi)^{3}} \int_{-\pi / d}^{\pi / d} \frac{d k_{z}}{\epsilon-\epsilon_{\alpha, n k_{z}+i \Gamma_{\alpha}}},
$$

where $\Delta S=2 \pi|e| B / \hbar c$ is the cross-sectional area in $\mathrm{k}$ space between the two successive Landau orbits,

$$
\epsilon_{\alpha, n k_{z}}=\Delta_{\alpha 0}+\omega_{\alpha}(n+1 / 2)+D_{\alpha} \cos j k_{z} d+g_{\alpha} \sigma \mu_{B} B,
$$

the energy dispersion for a present geometry of the Landau orbits, where $D_{\alpha}=2 t_{\alpha} J_{0}\left(j k_{f \alpha} d \tan \theta\right)$ with $J_{0}(x)$ the zeroth-order Bessel function, $\Gamma_{\alpha}=\pi / 2 \tau_{\alpha}, \tau_{\alpha}$ the scattering mean free time in zero field [16] in the band $\alpha$, and the background $(b g)$ is included in the DOS (3) too [6]. After integration we obtain an important exact result

$$
\begin{aligned}
\mathcal{N}_{\alpha}(\epsilon, B) & =\sum_{n=0}^{\infty} \rho_{\alpha} \omega_{\alpha} \nu\left(\epsilon-\epsilon_{\alpha, n}\right), \\
\nu\left(\epsilon-\epsilon_{\alpha, n}\right) & =\operatorname{Im} \frac{i}{\pi \sqrt{D_{\alpha}^{2}-\left(\epsilon-\epsilon_{\alpha, n}+i \Gamma_{\alpha}\right)^{2}}},
\end{aligned}
$$

where $k_{f \alpha}^{2} / 2 m_{\alpha}=\mu-\Delta_{\alpha}$,

$$
\epsilon_{\alpha, n}=\Delta_{\alpha 0}+\omega_{\alpha}(n+1 / 2)+g_{\alpha} \sigma \mu_{B} B,
$$

$\omega_{\alpha}=\left|B \cos \theta / m_{\alpha} c\right|$ the cyclotron frequency with the cyclotron mass $m_{\alpha}, \Delta_{\alpha 0}$ the band edge in zero magnetic field, $\mu$ the chemical potential, $g_{\alpha}$ the electron $g$-factor, $\sigma= \pm 1 / 2, \mu_{B}$ the Bohr magneton. The band index $\alpha \equiv b \sigma$ includes the band index $b$ and the spin index $\sigma$. There $\rho_{\alpha}$ is the zero-field density of states in the band $\alpha$. For the energies of interest, $\left|\epsilon-\Delta_{\alpha}\right|>2 t_{\alpha}$, $\rho_{\alpha}=m_{\alpha} / 2 \pi \hbar^{2} d$ per energy and unit volume, otherwise $\rho_{\alpha}=\left(m_{\alpha} / 2 \pi^{2} \hbar^{2} d\right) \arccos \left[\left(\epsilon-\Delta_{\alpha}\right) / 2 t_{\alpha}\right]$. Note that in a clean system at the magic angles, where $D_{\alpha}=\Gamma_{\alpha}=0$, the expression for $\nu$ reduces to $\nu\left(\epsilon-\epsilon_{\alpha, n}\right)=\delta\left(\epsilon-\epsilon_{\alpha, n}\right)$, meaning that the spectrum becomes $2 \mathrm{D}$, the Landau minibands reduce to the Landau levels, and the previous expressions [3] fully apply. The background density of states, $\rho_{b g}$, corresponding to possible non-quantized (or largely broadened) bands, can be included in (6) as the formal limit $\omega_{b g} \rightarrow 0$. Obviously, the non-quantized background will contribute to the non-oscillating characteristics of the system. In a clean limit the density of states takes a standard form with the one-dimensional square-root singularities $\mathcal{N} \sim 1 / \sqrt{D_{\alpha}^{2}-\left(\epsilon-\epsilon_{\alpha, n}\right)^{2}}$ [14].

By applying the Poisson formula [2] to the sum over $n$ in the thermodynamic potential

$$
\begin{aligned}
\Omega= & -T \sum_{n=0}^{\infty} \int d \epsilon \sum_{\alpha, b g} \rho_{\alpha} \omega_{\alpha} \nu\left(\epsilon-\epsilon_{\alpha, n}\right) \\
& \times \ln \left(1+\exp \frac{\mu-\epsilon}{T}\right)
\end{aligned}
$$

with $\mu_{\alpha}=\mu-\Delta_{\alpha}$ and $\Delta_{\alpha}=\Delta_{\alpha 0}+g_{\alpha} \sigma \mu_{B} B$, it can be written as

$$
\Omega=\Omega_{0}+\tilde{\Omega},
$$

where, after substituting $x=\left(\epsilon-\epsilon_{\alpha, n}\right) / D_{\alpha}$,

$$
\begin{aligned}
\Omega_{0}= & -T \int_{0}^{\infty} d \epsilon \sum_{\alpha, b g} \rho_{\alpha} \int_{-\infty}^{\infty} \frac{d x}{\pi} \operatorname{Im} \frac{i}{\sqrt{1-\left(x+i \Gamma_{\alpha}^{\prime}\right)^{2}}} \\
& \times \ln \left(1+\exp \frac{\mu_{\alpha}-\epsilon-D_{\alpha} x}{T}\right)
\end{aligned}
$$

is the "classical" part of the thermodynamic potential, with $\Gamma_{\alpha}^{\prime} \equiv \Gamma_{\alpha} / D_{\alpha}$. In the GCE $\Omega_{0}$ does not oscillate as a function of $1 / B$, and contains the contribution due to spin susceptibility (Pauli paramagnetism). At low temperatures one finds

$$
\Omega_{0}=-\sum_{\alpha, b g} \frac{1}{2} \rho_{\alpha}\left(\mu_{\alpha}^{2}+\frac{1}{2} D_{\alpha}^{2}\right) .
$$

The oscillating part of the thermodynamic potential includes only the Landau quantized bands,

$$
\begin{aligned}
\tilde{\Omega}= & -2 T \sum_{\alpha} \rho_{\alpha} \sum_{r=1}^{\infty} \int_{0}^{\infty} d \epsilon \int_{-\infty}^{\infty} \frac{d x}{\pi} \operatorname{Im} \frac{i}{\sqrt{1-\left(x+i \Gamma_{\alpha}^{\prime}\right)^{2}}} \\
& \times \ln \left(1+\exp \frac{\mu_{\alpha}-\epsilon-D_{\alpha} x}{T}\right) \cos 2 \pi r\left(\frac{\epsilon}{\omega_{\alpha}}-\frac{1}{2}\right) .
\end{aligned}
$$

This expression, after integrating over $\epsilon$ and $x$, reduces to

$$
\begin{aligned}
\tilde{\Omega} & =\frac{1}{24} \sum_{\alpha} \rho_{\alpha} \omega_{\alpha}^{2}+2 \sum_{\alpha} \sum_{r=1}^{\infty} A_{\alpha}^{\prime r} \cos 2 \pi r\left(\frac{F_{\alpha}}{B}-\frac{1}{2}-\psi_{\alpha}\right) \\
& =\frac{1}{24} \sum_{\alpha} \rho_{\alpha} \omega_{\alpha}^{2}+4 \sum_{b}^{\text {bands }} \sum_{r=1}^{\infty} A_{b}^{r} \cos 2 \pi r\left(\frac{F_{b}}{B}-\frac{1}{2}\right),
\end{aligned}
$$

where $F_{b}=\left(\mu-\Delta_{b 0}\right)\left|m_{b}\right| /\left(2 \mu_{B} m \cos \theta\right) \equiv \hbar c S_{f b} / 2 \pi e$, $S_{f b}$ is the mean Fermi surface zero-field cross-section (in standard units), and the spin-related phase $\psi_{\alpha}=\sigma \gamma_{b}$, $\gamma_{b}=g_{b}\left|m_{b}\right| /(2 m \cos \theta)$. If the scattering time were not dependent on the spin projection, the phase would be the only quantity explicitly depending on spin in Eq. (14), hence one can perform a summation over $\sigma$ in the second term to reveal the standard spin reduction factor $\cos \pi r \gamma_{b}$ [2].

The amplitudes of the Fourier harmonics in Eq. (14) are explicitly given by

$$
A_{b}^{r}=\frac{\rho_{b} \omega_{b}^{2}}{4 \pi^{2} r^{2}} R_{T}\left(\frac{2 \pi^{2} r T}{\omega_{b}}\right) R_{Y}\left(\frac{2 \pi r D_{b}}{\omega_{b}}\right) R_{D} R_{s},
$$

where $R_{T}(z)=z / \sinh z$ is the usual temperature reduction factor [2], $R_{Y}(z)=J_{0}(z)$ the orientation (Yamaji) factor noticed in [8], $R_{D}=e^{-2 \pi r \Gamma_{b} / \omega_{b}}$ the Dingle exponential damping due to collision broadening of the Landau minibands, $R_{s}=\cos \pi r \gamma_{b}$ the spin reduction factor, which all strongly depend on the orientation of the field, 


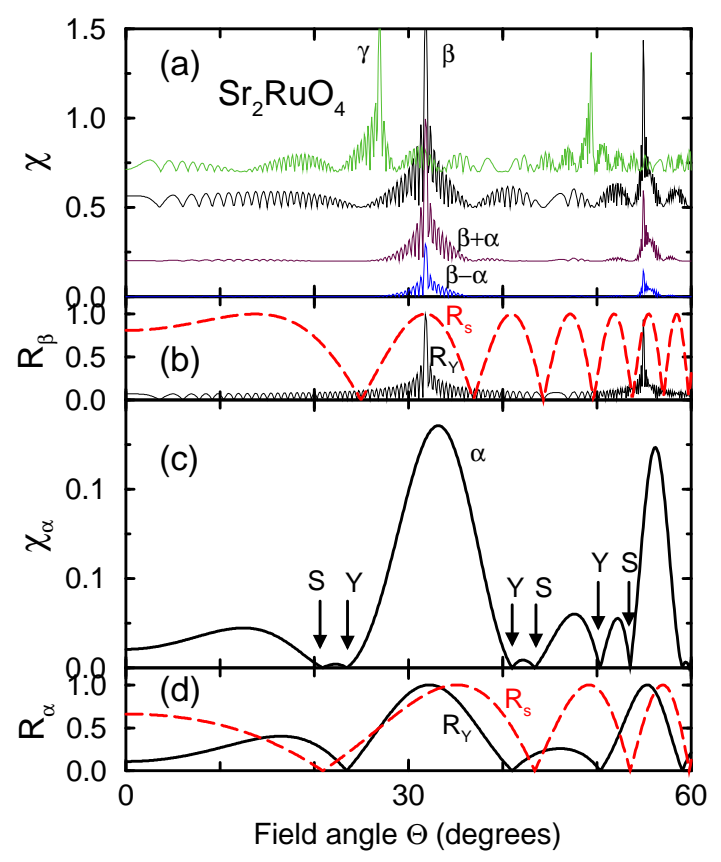

FIG. 1. The amplitudes of magnetic susceptibility harmonics $\chi_{\alpha}$ [panel (c)], $\chi_{\beta}, \chi_{\gamma}$, and $\chi_{\beta \pm \alpha}$ [panel (a)] in $\mathrm{Sr}_{2} \mathrm{RuO}_{4}$ for $T=T_{D}=0$. Parameters used in Eq.(5) are $d=6.37 \AA, j=2$ for $\alpha, j=1$ for $\beta$ and $\gamma$ bands [10]. The corresponding reduction factors $R_{Y}$ and $R_{s}$ are shown in panels (b) for $\beta$ and (d) for $\alpha$ bands. The arrows mark the dips in the harmonic $\chi_{\alpha}$, corresponding to zeros of the Yamaji $R_{Y}$ (Y) and the spin $R_{s}(\mathrm{~S})$ factors. The maximal amplitude of $\alpha, \beta$, and $\beta \pm \alpha$ bands is found at the first Yamaji angle $33^{\circ}$ (see text).

angle $\theta$ (Fig. 1). As usual, one can view the Dingle factor as corresponding to the effective temperature $T+T_{D}$ in a clean system, where the Dingle temperature $T_{D}=\Gamma_{b} / \pi$. For the magic angles, where $D_{\alpha}=0$, one has $R_{Y}=1$ and hence a pure 2D situation is recovered [3]. However, generally in a multiband case one should expect that only a spectrum of a particular band will become $2 \mathrm{D}$ at some particular magnetic field tilt angle.

The expression for $\tilde{\Omega}$, Eq.(14), contains the (first) term, responsible for the Landau diamagnetism and the oscillating term, which is responsible for the de Haas-van Alphen effect. It is small compared with the "classical" part, since $\tilde{\Omega} / \Omega_{0} \sim(\omega / \mu)^{2} \ll 1$ for the present semiclassical situation. The Fourier components appear with the frequencies $r F_{\alpha}$. We recover from $\Omega_{0}(12)$ and the first term in $\tilde{\Omega}$ (14) the Pauli $\chi_{P}=\frac{1}{4} \mu_{B}^{2} \sum_{\alpha} \rho_{\alpha} g_{\alpha}^{2}$ and the Landau $\chi_{L}=-\frac{1}{3} \mu_{B}^{2} \cos ^{2} \theta \sum_{\alpha} \rho_{\alpha}\left(m / m_{\alpha}\right)^{2}$ susceptibilities, respectively. Since usually $g_{\alpha}=2$, those are almost standard, apart from the $\cos ^{2} \theta$ factor characteristic of the present geometry with the tilted magnetic field.

Note that the chemical potential (and carrier density) oscillates in a closed system and, unlike in GCE, the "classical" part of $\Omega$ contributes to oscillations as well [3]. The relevant thermodynamic potential of the closed system (CE) is the free energy, $F=\Omega+\mu N$, for a fixed number of electrons, $N=-\partial \Omega / \partial \mu$. The chemical potential is [3]

$$
\begin{aligned}
& \mu=\mu_{0}+\tilde{\mu}, \\
& \tilde{\mu}=-\frac{\tilde{N}}{\rho} \equiv-\frac{\tilde{N}}{\rho_{q}+\rho_{b g}}
\end{aligned}
$$

where $\mu_{0}$ is the non-oscillating, while $\tilde{\mu}$ is the oscillating part of the chemical potential, $\tilde{N}=-\partial \tilde{\Omega} / \partial \mu$ is the oscillating part of the density of electrons, $\rho=\sum_{\alpha} \rho_{\alpha} \equiv$ $\rho_{q}+\rho_{b g}$ is the total density of states, including the quantized $\rho_{q}$ and the background $\rho_{b g}$ DOS. Substituting this expression into $\Omega_{0}$, Eq. (12), we obtain $F=F_{0}+\tilde{F}$, where the oscillating part is [3]

$$
\tilde{F}=\tilde{\Omega}-\frac{\tilde{N}^{2}}{2 \rho},
$$

while $F_{0}$ is the non-oscillating part. One sees that the difference between the free energies of the ensembles is directly proportional to the fluctuation of the particle density, as it should, and is suppressed by the background density of states $\rho_{b g}, \sim \tilde{N}^{2} /\left(\rho_{q}+\rho_{b g}\right)$. The oscillating part of the particle density is small, but both terms in (18) give comparable contribution to the magnetization oscillations. Indeed, $\tilde{N}=\sum_{\alpha} \tilde{N}_{\alpha}$, and at low temperatures

$$
\frac{\tilde{N}_{\alpha}}{N_{0}} \sim \frac{\rho_{\alpha}}{\rho} \frac{B}{F_{\alpha}} \ll 1, \quad T<\omega_{\alpha},
$$

since $F_{\alpha} \gg B$, and it falls off exponentially with temperature, $\tilde{N}_{\alpha} / N_{0} \sim(T / \mu) \exp \left(-2 \pi^{2} T / \omega_{\alpha}\right)$, at $T>\omega_{\alpha}$. Since the density oscillations are small, sometimes they can be screened by the back electrode in e.g. quantum well structures [9]. In a more explicit form one obtains

$$
\begin{aligned}
\tilde{F}= & \frac{1}{24} \sum_{\alpha} \rho_{\alpha} \omega_{\alpha}^{2}+4 \sum_{b} \sum_{r=1}^{\infty} A_{b}^{r} \cos 2 \pi r\left(\frac{F_{b}}{B}-\frac{1}{2}\right) \\
& -16 \sum_{b, b^{\prime}} \sum_{r, r^{\prime}=1}^{\infty} C_{b b^{\prime}}^{r r^{\prime}} \sin 2 \pi r\left(\frac{F_{b}}{B}-\frac{1}{2}\right) \sin 2 \pi r^{\prime}\left(\frac{F_{b^{\prime}}}{B}-\frac{1}{2}\right) .
\end{aligned}
$$

It is the last term, which yields the combination Fourier harmonics with the frequencies $F=r F_{b} \pm r^{\prime} F_{b^{\prime}}$. Their amplitudes,

$$
C_{b b^{\prime}}^{r r^{\prime}}=\frac{2 \pi^{2} r r^{\prime} A_{b}^{r} A_{b^{\prime}}^{r^{\prime}}}{\left(\rho_{q}+\rho_{b g}\right) \omega_{b} \omega_{b^{\prime}}}
$$

are comparable with the standard single-band harmonics at low temperatures, $T<\omega_{\alpha} / 2 \pi^{2} r$, as found earlier [3] and confirmed experimentally 9 11]. The combination harmonics are suppressed in presence of the background density of states. The spin factor depends on the tilt angle, and this results in a strong angular dependence of the corresponding dHvA amplitudes [10,11]. Incidentally, 
if one of the angular dependent factors vanishes for some particular harmonic, $r_{0} F_{b}$, it would not mix up with other bands to produce combination harmonics, whereas $r F_{b}$ $\left(r \neq r_{0}\right)$ would (cf. numerical results [17]).

Similar to the usual 3D situation, the oscillations produce the (partial) contributions to magnetization, $M_{\alpha}$, and susceptibility, $\chi_{\alpha}$, which are much larger than the non-oscillating contributions. Indeed, the ratio of corresponding amplitudes at low temperatures is $\left|\chi_{\alpha}^{r}\right| / \chi_{0} \sim$ $\left(F_{\alpha} / B\right)^{2}\left(\rho_{\alpha} m / \pi^{2} \rho\left|m_{\alpha}\right| r\right) J_{0}\left(2 \pi r D_{\alpha} / \omega_{\alpha}\right) e^{-2 \pi r \Gamma_{\alpha} / \omega_{\alpha}} \gg$ 1 , since $F_{\alpha} / B \gg 1$. Proportionality to $\left(F_{\alpha} / B\right)^{2}$ is the property of the two-dimensional geometry [2]. The amplitudes of the standard and the combination harmonics can be easily found from the expressions given above. The ratio of susceptibilities is

$$
\frac{\chi_{r F_{b}, \pm r^{\prime} F_{b^{\prime}}}^{c}}{\chi_{r F_{b}}^{\mu}}=\frac{8 \pi^{2} r r^{\prime} A_{b^{\prime}}^{r^{\prime}} \cos \pi r^{\prime} \gamma_{b^{\prime}}}{\rho \omega_{b} \omega_{b^{\prime}}}\left(\frac{F}{r F_{b}}\right)^{2}
$$

with $F=r F_{b} \pm r^{\prime} F_{b^{\prime}}$. Thus, for $r=r^{\prime}=1$ we have, for a warped 2-band cylindrical Fermi surface without the background DOS,

$$
\begin{aligned}
\frac{\chi_{F_{b}, \pm F_{b^{\prime}}}^{c}}{\chi_{F_{b}}^{\mu}}= & \frac{4 \pi^{2} m_{b^{\prime}}}{m_{b}+m_{b^{\prime}}} \frac{T J_{0}\left(2 \pi D_{b^{\prime}} / \omega_{b^{\prime}}\right) \cos \pi \gamma_{b^{\prime}} e^{-2 \pi \Gamma_{b^{\prime}} / \omega_{b^{\prime}}}}{\omega_{b} \sinh \left(2 \pi^{2} T / \omega_{b^{\prime}}\right)} \\
& \times\left(\frac{F_{b} \pm F_{b^{\prime}}}{F_{b}}\right)^{2},
\end{aligned}
$$

where $\chi^{\mu}$ denotes the amplitude of the standard dHvA harmonic for an open system, with $\chi^{c}$ the amplitude of the novel combination harmonics. We see that the combination harmonics are suppressed exponentially with respect to temperature [3] and/or disorder compared to the standard harmonics, Fig. 2. In addition, they contain the product of the angular dependent reduction factors and, therefore, vary rapidly with the angle $\theta$, their frequency being dominated by the band with the largest ratio $2 t_{b} / \omega_{b}$ (e.g. $\beta$-band in $\beta \pm \alpha$ harmonics in $\mathrm{Sr}_{2} \mathrm{RuO}_{4}$, Fig. 1).

It is important to note that even in a simplest case of a one-band system the standard harmonics $r F_{b}$ are modified by the second quadratic term, therefore

$$
\begin{aligned}
& \chi_{r F_{b}}^{N}=\chi_{r F_{b}}^{\mu}+\sum_{p=1}^{r-1} \chi_{(r-p) F_{b}, p F_{b}}^{c}+\sum_{p=1}^{\infty} \chi_{(r+p) F_{b},-p F_{b}}^{c}, \\
& \chi_{r F_{b} \pm r^{\prime} F_{b^{\prime}}}^{N}=\chi_{r F_{b}, \pm r^{\prime} F_{b^{\prime}}}^{c}, \quad b \neq b^{\prime},
\end{aligned}
$$

and the wavefront of magnetization of the main harmonics in the closed system with the carrier density $N=$ const is inverted compared to the open system, $\mu=$ const, as it should [2,4,6].

The present formalism is applied to $\mathrm{Sr}_{2} \mathrm{RuO}_{4}$ with the results shown in Figs. 1 and 2, calculated with the parameters from Refs $[10,5]$ and the g-factor $g_{b}=2$ for all the bands. The main maxima in the Fourier harmonics of

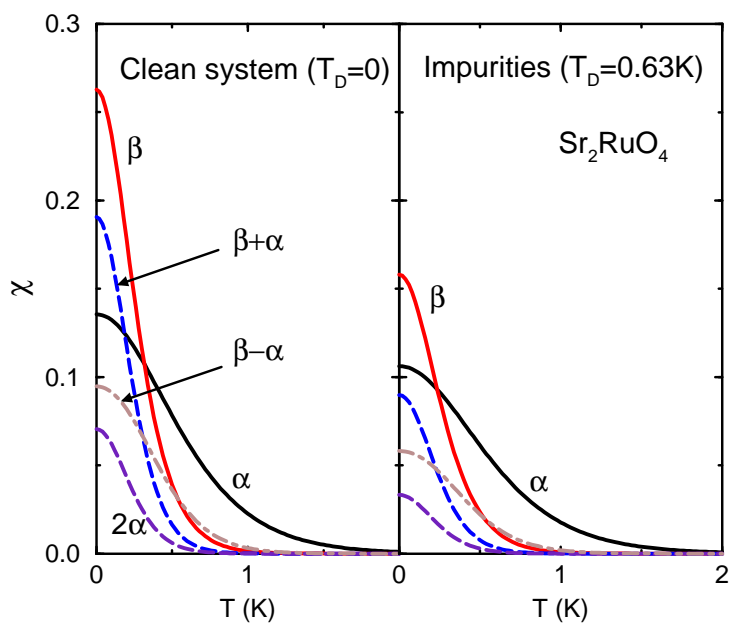

FIG. 2. The amplitudes of the harmonics in Fig. 1, including also $2 \alpha$, versus the temperature in a clean (left panel) and a disordered (right panel) $\mathrm{Sr}_{2} \mathrm{RuO}_{4}$ at the (Yamaji) angle $\theta=33^{\circ}$. The Dingle temperature $T_{D}=0.63 \mathrm{~K}[10]$.

susceptibility $\chi$ for the band $\alpha$ are found at $\theta_{\alpha 1(2)}=33^{\circ}$ $\left(55^{\circ}\right)$, for the band $\beta$ at $\theta_{\beta} \approx \theta_{\alpha}$, and for band $\gamma$ at $\theta_{\gamma 1(2)}=27^{\circ}\left(49^{\circ}\right)$, which is in fair agreement with the data $\theta_{\alpha 1}=30.6^{\circ}, \theta_{\beta 1}=30^{\circ}$ and $\theta_{\gamma 1}=15.3^{\circ}$, Ref. [10], and $\theta_{\alpha 1}=30^{\circ}, \theta_{\beta 1(2)}=26^{\circ}\left(56^{\circ}\right)$, Ref. 11]. Those $\max$ ima in the harmonics apparently coincide with the points where the Yamaji factor is unity, $R_{Y}=1$, and the system effectively becomes 2D, Figs. 1(b),(d). The minima, observed in $\alpha$ band at $24^{\circ}$ 10] (25 [11]) and $40^{\circ}$, are very close to the points were both the Yamaji $R_{Y}$ and the spin $R_{s}$ factors vanish: $R_{Y}=0$ at $23^{\circ}$ and $41^{\circ}$, and $R_{s}=0$ at $21^{\circ}$ and $43^{\circ}$, Fig. $1(\mathrm{~d})$.

The conspicuous rapid variation with the field angle $\theta$ of the main, $\chi_{\beta}, \chi_{\gamma}$, and the combination, $\chi_{\beta-\alpha}$, $\chi_{\beta-\alpha}$, harmonics, Fig.1(a), is explained by the large factor $2 t_{b} / \omega_{b}$ in the argument of the Bessel function in the Yamaji factor, Eqs.(15), (23): $2 t_{\beta} / \omega_{\beta}=7.5$, $2 t_{\gamma} / \omega_{\gamma}=5$. This necessarily leads to the high frequency variations of the amplitudes, and apparently only the envelope of those oscillations has been sampled experimentally in Refs. 10,11] (see also a numerical study [8]). Those rapid variations with angle $\theta$ might be observable, since the condition for the Yamaji approximation, $\pi k_{f b}^{2}\left(t_{b} / \epsilon_{f}\right)^{2} \ll \Delta S$ [13] seems to hold for the $\beta$-band $\left[\pi k_{f \beta}^{2}\left(t_{\beta} / \epsilon_{f}\right)^{2}: \Delta S \approx 1: 5\right]$, while for the $\gamma$-band the ratio is only about $1: 3$. Interestingly, the spin-factor $R_{s}$ defines the envelope of the $\beta$-amplitude, cf. Figs. 1(a) and 1(b), so the studies of the minima and maxima on the $\chi(\theta)$ [10] should allow for accurate determination of the g-factors $g_{b}$.

The combination harmonics contain the extra temperature and the Dingle reduction factors, so they are falling off with either temperature or disorder, or both, faster than the standard harmonics do, see Eqs.(21), (15), Fig.2. 
The temperature dependence of the combination harmonics generally cannot be characterized by some effective mass $m_{r b, \pm r^{\prime} b^{\prime}}$, although at higher temperatures the relation $m_{r b, \pm r^{\prime} b^{\prime}} \approx r m_{b}+r^{\prime} m_{b^{\prime}}$ holds approximately. All the harmonics decay quickly with $T$, especially in the presence of even weak disorder (the Dingle temperature $T_{D}=0.63 \mathrm{~K}[10]$ ), Fig. 2(b), which is in very good agreement with experiment (cf. Fig. 2 in Ref. [5]).

Importantly, in the first experiments on $\mathrm{Sr}_{2} \mathrm{RuO}_{4}$ the disorder was actually larger, since the elastic mean free path was $l \sim 10^{3} \AA[5]$, compared to $l \sim 2050-5000 \AA$ in the later experiments [10,11]. The higher disorder in the first samples, and relatively small magnitude of the combination peaks at the field $B$ directed along the c-axis, $\theta=0$, instead of the "magic" angle $\theta=30^{\circ}$, where all the amplitudes are enhanced, Fig. 1, has possibly prevented the discovery of the combination harmonics, predicted in Ref. [4], in the first experiments on $\mathrm{Sr}_{2} \mathrm{RuO}_{4}$ [5]. The combination harmonics have been detected in Refs. [9 11. The dependence of the $\mathrm{dHvA}$ combination amplitudes on angle, temperature, and disorder provide additional valuable tool for studying the band structure and carrier densities in the multiband quasi-two-dimensional metals.

[1] S. Uji et al., Nature 410, 908 (2001).

[2] D. Shoenberg, Magnetic Oscillations (Cambridge Univ. Press, Cambridge, 1984).

[3] A.S. Alexandrov and A.M. Bratkovsky, Phys. Rev. B 63, 033105 (2001).

[4] A.S. Alexandrov and A.M. Bratkovsky, Phys. Rev. Lett. 76, 1308 (1996).

[5] A.P. Mackenzie et al., Phys. Rev. Lett. 76, 3786 (1996).

[6] A.S. Alexandrov and A.M. Bratkovsky, Phys. Lett. A 234, 53 (1997).

[7] M. Nakano, J. Phys. Soc. Jpn. 6619 (1997).

[8] M. Nakano, J. Phys. Soc. Jpn. 68, 1801 (1999); Phys. Rev. B 62, 45 (2000).

[9] R.A. Shepherd, M. Elliott, W.G. Herrenden-Harker, M. Zervos, P.R. Morris, M. Beck, and M. Ilegems, Phys. Rev. B60, R11277 (1999).

[10] Y. Yoshida et al., J. Phys. Soc. Jpn. 68, 3041 (1999); ibid., 67, 1677 (1998).

[11] E. Ohmichi, Y. Maeno, and T. Ishiguro, J. Phys. Soc. Jap. 68, 24 (1999); E. Ohmichi et al., Phys. Rev. B 59, 7263 (1999).

[12] M.V. Kartsovnik, P.A. Kononovich, V.N. Laukhin, and I.F. Shchegolev, Pisma Zh. Eksp. Teor. Fiz. 48, 498 (1988).

[13] K. Yamaji, J. Phys. Soc. Jpn. 58, 1520 (1989).

[14] V.N. Lutsky, M.I. Kaganov, and A.Ya. Shik, Zh. Eksp. Teor. Fiz. 92 (1987) 721.

[15] R.B. Dingle, Proc. Roy. Soc. A211, 517 (1952).

[16] Yu. A. Bychkov, JETP 39, 977 (1961) [Zh. Eksp. Teor.
Fiz. 12, 977 (1961)].

[17] K. Kishigi, Y. Hasegawa, and M. Miyazaki, J. Phys. Soc. Jpn. 69, 821 (2000). 